Artigo Original

\title{
Efeito protetor após sessões de exercício excêntrico: comparação entre membros superiores e inferiores ${ }^{1}$
}

\author{
Leonardo Coelho Rabello de Lima \\ Benedito Sérgio Denadai
}

\author{
Instituto de Biociências. UNESP - Universidade Estadual Paulista, Campus de Rio Claro, \\ Departamento de Educação Física, Laboratório de Avaliação da Performance Humana \\ (LAPH) , Rio Claro, SP, Brasil
}

\begin{abstract}
Resumo: O objetivo foi comparar a magnitude do efeito protetor (EP) contra o dano muscular (DM) induzido por uma sessão de exercícios excêntricos (EEM) entre os extensores do joelho e os flexores do cotovelo. Doze sujeitos do gênero masculino foram divididos em 2 grupos, braços (GB) e pernas (GP), e realizaram 2 sessões de EEM. Foram coletados 3 marcadores de DM, sendo eles, pico de torque isométrico (PTI), creatina quinase (CK) e percepção subjetiva de dor (PSD), antes, imediatamente após (com exceção da CK) e 48 horas após cada sessão de EEM. Foi encontrada queda significante de PTI e aumento significante de CK e PSD tanto imediatamente e 48 horas após a primeira sessão de EEM para o GB. No GP houve aumento significante de CK 48 horas após os EEM e da PSD imediatamente após os EEM, decorrentes da primeira sessão. No GB, a segunda sessão apenas provocou queda de PTI imediatamente após os EEM, enquanto no GP houve aumento significante apenas na PSD imediatamente após a segunda sessão de EEM. Apenas a CK apresentou EP para ambos os grupos. Pudemos concluir que o EP foi maior para o GB em comparação com o GP. Esse fenômeno pode ter ocorrido em detrimento da existência de um EP prévio para o GP, uma vez que este membro realiza contrações excêntricas intensas com maior freqüência no dia-a-dia, quando comparados com os GB.
\end{abstract}

Palavras-chave: Dano muscular. Fator de proteção. Contrações excêntricas.

\section{The repeated bout effect: a comparison between upper and lower limbs}

\begin{abstract}
The objective was to compare the magnitude of the repeated bout effect (RBE) induced by an eccentric exercise session (EES) between the knee extensors and elbow flexors. Twelve male subjects were divided into two groups, arms (AG) and legs (LG), and executed two EES. We collected three markers of muscle damage, they were, isometric peak torque (IPT), creatine kinase (CK) and subjective perception of pain (PSD) before, immediately after (except CK) and 48 hours after each EES. Significant decrease in PTI was found as well as significant increase in CK and PSD both immediately and 48 hours after the first EES for the AG. In the LG there was a significant increase in CK 48 hours after, and PSD immediately after the first EES. In the AG, the second EES caused a drop of only PTI immediately after it, while the LG presented a significant increase only in PSD immediately after the second EES. RBE was found only for CK in both groups. We concluded that the RBE was higher in the AG compared to the LG. This phenomenon may have occurred over the existence of a protection for the $L G$, since this member performs intense eccentric contractions more frequently in day-to-day, compared to AG.
\end{abstract}

Key-words: Muscle damage. Repeated Bout Effect. Eccentric contractions.

\section{Introdução}

Um dos principais focos de estudos sobre treinamento de força tem sido o dano muscular (DM), sendo esse caracterizado por microlesões sofridas pelo tecido muscular por contrações intensas e, geralmente, de caráter excêntrico. A contração excêntrica é a que causa maior índice de dano muscular, pois, para este tipo de contração, é recrutado um baixo número de unidades motoras, o que gera elevado estresse mecânico sobre as fibras musculares das unidades recrutadas (MALM et al., 1999). Essa

\footnotetext{
Derivado do Trabalho de Conclusão de Curso da Universidade Estadual Paulista Julio de Mesquita Filho Campus de Rio Claro, no ano de 2010.
}

elevada tensão por área de secção transversa (AST) da fibra muscular pode induzir diversos processos mecânicos como a desorganização da linha $Z$ dos sarcômeros, assim como a perda de mitocôndrias e rupturas ou modificações estruturais nos miofilamentos de miosina (CLARKSON E HUBAL, 2002). Diversas variáveis são coletadas no diagnóstico e cálculo da magnitude do DM, sendo consideradas como principais a queda da capacidade de produção de força por meio de contrações voluntárias máximas (CVM), o extravasamento da enzima creatina quinase $(\mathrm{CK})$ do tecido muscular para o sangue, a percepção subjetiva de dor (PSD) decorrente de processo inflamatório e a 
diminuição da amplitude de movimento (ADM)

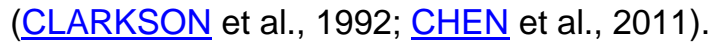

Boa parte dos estudos na área têm se voltado para a manipulação de variáveis para a elaboração de protocolos de exercícios excêntricos que induzam DM de maior magnitude possível. Tem sido verificado que menores tempos de recuperação entre séries (MAYHEW et al., 2005), maiores velocidades angulares durante as contrações (CHAPMAN et al., 2006) e maiores amplitudes de movimento (NOSAKA E SAKAMOTO, 2001) determinam aumento do DM.

Já está bem descrito na literatura que após o DM, o organismo se reestrutura e promove a recuperação da musculatura afetada gerando, assim, uma proteção parcial contra eventuais situações de estresse em que possa ocorrer o DM novamente (CLARKSON E HUBAL, 2002; NOSAKA et al.,2005a; NOSAKA et al., 2005b; HOWATSON et al., 2007), ou seja, haverá uma atenuação nos sintomas do DM quando realizada uma segunda sessão de mesmo volume e intensidade. Este fenômeno é conhecido como efeito protetor (EP) decorrente de sessões de exercício excêntrico e pode durar entre 6 e 9 meses (NOSAKA et al., 2001a).

É entendido que uma primeira sessão de exercícios excêntricos máximos (EEM) não precisa causar grandes danos para que haja um fator de proteção. Na verdade, dez, seis, ou até mesmo duas contrações excêntricas máximas são capazes de conferir um efeito protetor (EF) significativo (NOSAKA et al., 2001b). O EF é específico para 0 músculo trabalhado excentricamente sem evidências de proteger, também, outros grupos não relacionados. Porém, a proteção não parece ser necessariamente conferida para apenas um tipo de exercício, podendo proteger um mesmo grupo muscular contra o DM em diferentes práticas esportivas e/ou tipos de contração (ESTON et al., 1996).

Por mais conhecidas que sejam as condições para induzir o DM, gerando assim um EP, pouco se conhece sobre os mecanismos responsáveis pela ocorrência deste. Diversas teorias foram formuladas para explicar a ocorrência do EP e, em uma revisão realizada por McHugh (2003) elas foram divididas em três grandes grupos: adaptações neurais, mecânicas e celulares. As teorias que apontam adaptações neurais como responsáveis pelo $E P$, se valem de recursos como a eletromiografia para a determinação da ativação de unidades motoras (UM). Essas teorias têm mostrado que, após a ocorrência do $\mathrm{DM}$, o sistema nervoso passa a recrutar maior número de fibras de contração lenta, além de aumentar a sincronia no recrutamento das UM (WARREN et al., 2000; McHUGH et al., 2001).

Já as teorias que levam em conta adaptações mecânicas decorrentes do DM, dizem que o aumento do stiffness passivo e ativo do músculo é um importante processo de adaptação para o EP (POUSSON et al.,1990), também considerando o aumento do tecido conjuntivo como uma proteção mecânica eficiente contra o DM (LAPIER et al., 1995). Além desses mecanismos apresentados, também se demonstra que, após a recuperação do tecido muscular danificado, há um aumento do conteúdo da proteína desmina nas miofibrilas, sendo essa uma das responsáveis pela orientação longitudinal e horizontal dos sarcômeros (BARASH et al., 2002).

Por fim, a teoria que leva em conta a adaptação celular como principal fator de proteção contra o DM sugere que um dos motivos da proteção é o aumento do número de sarcômeros em série na fibra muscular, o que diminuiria a tensão por unidade de sarcômero, gerando menor estresse sobre as microestruturas do tecido muscular (LYNN E MORGAN, 1994; LYNN et al., 1998, BROCKETT et al., 2001). Também dentro das adaptações celulares, há estudos demonstrando que um fortalecimento do retículo sarcoplasmático seria responsável pelo $E P$, uma vez que o DM compromete o acoplamento excitação-contração (CLARKSON E TREMBLAY, 1988).

Uma parcela de estudos mais atuais vem investigando e comparando as respostas de diferentes grupamentos musculares à atividade excêntrica máxima. Jamurtas et al. (2005) investigaram a diferença da magnitude do DM causado nos extensores do joelho (EJ) e flexores do cotovelo (FC) por sessões de exercício excêntrico de mesma intensidade relativa. Neste estudo, os voluntários realizaram protocolos de contrações excêntricas submáximas $(75 \%$ do pico de torque isocinético excêntrico) isocinéticas $\left(60^{\circ} . \mathrm{s}^{-1}\right)$ para ambos os grupos musculares, em ordem aleatória, com intervalo de 14 dias entre sessões para que houvesse recuperação completa do tecido muscular. Os resultados demonstraram aumentos significativamente menores da atividade sérica de $\mathrm{CK}$ e menor perda 
de força muscular para os EJ quando comparados com os FC, porém não foram encontradas diferenças significativas entre os músculos para as mudanças de ADM e PSD. Já Saka et al. (2009) demonstraram que diminuições no PTI e na ADM, assim como aumentos da atividade sérica de $\mathrm{CK}$, concentração de mioglobina $(\mathrm{Mb})$ no sangue, e da PSD foram significativamente menores para os EJ quando comparados com FC após a realização de 3 séries de 15 repetições isocinéticas $\left(30^{\circ} . \mathrm{s}^{-1}\right)$ excêntricas máximas de mesma $\operatorname{ADM}\left(90^{\circ}\right)$. As justificativas dadas pelos autores para tais achados foram que contrações excêntricas estão mais presentes em situações cotidianas para os EJ do que para os FC, e isso geraria um EP parcial para tal grupamento, além de existirem diferenças na curva de tensão-comprimento, arquitetura muscular e tipos de fibras entre os músculos.

Em um estudo mais amplo, Chen et al. (2011) compararam o DM induzido por 5 séries de 6 contrações isocinéticas $\left(90^{\circ} . \mathrm{s}^{-1}\right)$ excêntricas máximas de mesma $\operatorname{ADM}\left(90^{\circ}\right)$ entre quatro diferentes grupamentos musculares: extensores (EJ) e flexores (FJ) do joelho, e extensores (EC) e flexores (FC) do cotovelo. Os resultados obtidos demonstraram que os EC e FC responderam de maneira similar ao exercício, não apresentando diferenças significativas entre as alterações dos marcadores de DM coletados. Os FJ apresentaram maior proteção contra o DM em relação aos EF e EC e os EJ foram os músculos menos afetados pela sessão de exercícios excêntricos, confirmando os resultados dos estudos anteriores. Porém, este estudo demonstrou que o fator de proteção não é dependente unicamente do membro, uma vez que os EJ e FJ apresentaram respostas diferentes.

Tendo em mente que os estudos acima citados investigam apenas a expressão do DM entre diferentes grupos musculares, o objetivo do presente estudo foi identificar se também há diferenças entre o FP contra o DM conferido por uma sessão de exercícios isocinéticos excêntricos máximos entre dois grupos musculares. Neste estudo testou-se a hipótese de que o FP seria menor para os EJ do que para os FC.

\section{Material e Métodos}

\section{Sujeitos}

Participaram do estudo 12 estudantes universitários do sexo masculino, aparentemente saudáveis e sem experiência com treinamento resistido nos 6 meses que antecederam o período de coletas. Todos os sujeitos leram e assinaram duas vias de um Termo de Consentimento Livre e Esclarecido aprovado pelo comitê de ética da instituição e ficaram com uma delas para si. As médias de massa, idade e altura foram 79,8 \pm $15,1 \mathrm{~kg}, 21,4 \pm 1,6$ anos e $173 \pm 6 \mathrm{~cm}$, respectivamente. Os sujeitos foram randomicamente distribuídos em dois grupos, braços (GB) (N=6) e pernas (GP) $(\mathrm{N}=6)$, baseados no segmento corporal a ser examinado no estudo. Não houve diferenças significativas entre idade, altura e massa corporal entre os grupos. Todos os sujeitos foram aconselhados a não realizar nenhum tipo de exercício intenso, mudança radical na dieta $e$ ingesta de suplementos alimentares e/ou medicamentos antiinflamatórios durante o período de coletas.

\section{Design Experimental}

Todos os sujeitos compareceram ao laboratório por cinco vezes no total. A primeira visita foi designada para familiarização ao dinamômetro isocinético (Biodex Pro 3 System, Shirley, NY, USA) e ao ambiente de coleta, além da explicação dos procedimentos experimentais a serem adotados nas coletas seguintes e fornecimento do consentimento para participar da pesquisa. A segunda visita foi planejada para a realização de uma primeira sessão de EEM. Antes de realizarem o EEM, os sujeitos realizaram aquecimento da musculatura a ser trabalhada e eram coletados os marcadores CK, PTI e PSD. Imediatamente após a realização dos exercícios excêntricos, eram coletados apenas os marcadores PTI e PSD, uma vez que alterações significativas no marcador $\mathrm{CK}$ não ocorrem imediatamente após o EEM (CLARKSON E HUBAL, 2002). Quando passadas 48 horas da realização do EEM, os sujeitos realizavam a terceira visita ao laboratório para a monitoração da magnitude do DM por meio de coleta de CK, PTI e PSD. A escolha pelo intervalo de 48 horas foi baseada em uma concordância nas alterações dos três marcadores coletados que, neste período, costumam apresentar a maior diferença em relação à condição basal (NOSAKA et al., 2001b; JAMURTAS et al., 2005). 
Depois de passados 14 dias da primeira sessão de exercícios excêntricos, era assumido que todos os sujeitos já haviam se recuperado do DM conferido pela primeira sessão (NOSAKA et al., 2001b; HOWATSON et al., 2007) e eles retornavam ao laboratório para a execução do mesmo protocolo de EEM realizado na segunda visita. Após 48 horas da sessão de EEM, os sujeitos voltavam novamente ao laboratório para que os procedimentos de coleta realizados na terceira visita fossem repetidos. $O$ delineamento experimental está representado na Figura 1.

\begin{tabular}{|c|c|c|c|c|c|c|c|}
\hline 1ㄹ Visita & 2a visita & $48 \mathrm{~h}$ & 3 a visita & 14 Dias & 4a visita & $48 \mathrm{~h}$ & 5a visita \\
\hline Esclarecimentos & $\begin{array}{l}C K \\
P T I \\
P S D\end{array}$ & 尽 & - & 疍 & $\begin{array}{l}C K \\
\text { PTI } \\
\text { PSD }\end{array}$ & 尽 & - \\
\hline Familiarizações & EEM & 尽 & $\begin{array}{c}C K \\
P T I \\
P S D\end{array}$ & 尽 & EEM & 尽 & $\begin{array}{c}C K \\
P T I \\
P S D\end{array}$ \\
\hline Consentimento & $\begin{array}{l}\text { PTI } \\
\text { PSD }\end{array}$ & 尽 & - & 层 & $\begin{array}{l}\text { PTI } \\
\text { PSD }\end{array}$ & 尽 & - \\
\hline
\end{tabular}

Figura 1. Delineamento experimental com as visitas realizadas ao laboratório e os procedimentos adotados. As siglas em itálico representam coletas realizadas.

\section{Exercício Excêntrico}

Os EEM foram realizados no dinamômetro isocinético supracitado. Para o posicionamento dos sujeitos de ambos os grupos, foram seguidas as orientações fornecidas pelo fabricante. Todos os sujeitos tiveram as medidas referentes ao seu posicionamento no dinamômetro anotadas na primeira visita, para que fossem utilizadas durante o delineamento experimental, garantindo, assim, a reprodutibilidade das medidas (DVIR, 2002). $O$ protocolo de EEM foi composto por 4 séries de 12 contrações excêntricas máximas em uma amplitude articular de $90^{\circ}\left(10^{\circ}-100^{\circ}\right.$ para o GP e $90^{\circ}-0^{\circ}$ para $\circ \mathrm{GB}$, sendo considerado $0^{\circ}$ como extensão completa) a uma velocidade angular de $180^{\circ} . \mathrm{s}^{-1} \mathrm{com} 60$ segundos de intervalo entre séries. A fase concêntrica das contrações foi realizada de maneira passiva a $60^{\circ} \cdot \mathrm{s}^{-1}$, com ajuda do examinador.

Anteriormente à realização do EEM, os sujeitos foram orientados a produzir o máximo de força possível contra o movimento do braço de alavanca do dinamômetro. Todas as contrações foram realizadas sob supervisão de um único examinador que mantinha um padrão uniforme de incentivo (DVIR, 2002). Durante as contrações foi fornecido feedback visual para os sujeitos por meio de um gráfico de barras no display do dinamômetro para que esses tentassem sempre alcançar ou superar a força gerada na contração anterior.

\section{Marcadores}

Três marcadores indiretos de DM foram selecionados com base no que se tem utilizado em outros estudos (CLARKSON e HUBAL, 2002; McHUGH, 2003). Todos os procedimentos de coleta foram realizados na mesma ordem e pelo mesmo examinador.

A atividade sérica da enzima creatina quinase (CK) foi calculada por meio de coleta de $500 \mu \mathrm{l}$ de sangue do lóbulo da orelha dos sujeitos. Para que essa amostra fosse coletada sem a ocorrência de hemólise, foi utilizada uma pomada vasodilatadora (Finalgon, Pharma $\mathrm{GmbH} \& \mathrm{Co}$. $K G)$. Depois de obtida a amostra, o sangue era centrifugado por 6 minutos a $5600 \mathrm{rpm}$ em uma centrífuga (Centrífuga Microhemato Modelo 2410, Fanem, São Paulo, Brasil) e então o soro obtido era analisado em um espectofotômetro (Bio-2000, Bioplus, São Paulo, Brasil) com a utilização de um kit comercial de análise de CK (CK-NAC UV, Wiener Lab., Rosário, Argentina). A variação normal na concentração de CK no soro sanguíneo para homens adultos, utilizando este método, é de 24 a $195 \mathrm{U} / \mathrm{l}$.

Para a quantificação da percepção subjetiva de dor (PSD), os voluntários foram orientados a palpar a musculatura a ser utilizada durante 0 protocolo e preencher uma escala de análogos visuais (EAV) ( $\underline{\mathrm{CHEN}}$ et al., 2007). A EAV consistia de uma linha continua de $100 \mathrm{~mm}$ com 
os dizeres "Sem dor" em um lado $(0 \mathrm{~mm})$, e "Muita, muita dor" no outro (100mm).

$\mathrm{O}$ pico de torque isométrico ( $\mathrm{PTI}$ ) foi medido por meio de 3 contrações voluntárias máximas isométricas (CVMI) realizadas no dinamômetro isocinético supracitado. Assim como durante o EEM, as recomendações do fabricante foram seguidas para o posicionamento dos sujeitos e reprodutibilidade das medidas. As $3 \mathrm{CVMI}$ foram realizadas a $30^{\circ}$ para o GB ( $\underline{\mathrm{CONNOLLY}}$ et al., 2006) e a $60^{\circ}$ para o GP (SAKA et al., 2009), com duração de 3 segundos cada e 60 segundos de intervalo entre elas. O PTI foi calculado como a média dos PTI das 3 CVMI (NOSAKA et al., 2007).

\section{Tratamento Estatístico}

Os dados estão expressos em média \pm erro padrão. Mudanças em todos os marcadores em relação ao tempo foram comparadas entre a primeira e segunda sessões para cada grupo utilizando ANOVAs two-way $2 \times 3$ (sessões $\mathrm{x}$ tempo) para medidas repetidas. Nas situações em que a ANOVA demonstrou efeito significante, foi empregado o post hoc de Tuckey para identificar diferenças entre as médias nas diferentes sessões. Quando constatado EP para o mesmo marcador nos dois grupos, foi calculado um fator de proteção (FP) para cada sujeito. O cálculo de FP consistiu em, primeiramente relativizar os valores de cada um dos marcadores em função de percentuais do valor basal. Após isso, para PTI, o valor obtido na segunda sessão era subtraído deste mesmo valor na primeira sessão, enquanto para CK e PSD, o valor obtido na primeira sessão era subtraído deste mesmo valor na segunda sessão, uma vez que as cinéticas para esses marcadores apresentam comportamentos diferentes. Os FPs obtidos para GB e GP foram comparados por meio de testes não paramétricos de Mann-Whitney para amostras independentes. O nível de significância adotado foi de $p \leq 0,05$.

\section{Resultados}

Não foram encontradas diferenças significativas entre os valores basais das duas sessões de EEM para ambos os grupos.

\section{Atividade sérica de CK}

O GB apresentou diferença significativa ( $p=$ 0,03 ) apenas entre o valor basal referente à primeira sessão de EEM $(111,5 \pm 32,37 \mathrm{U} / \mathrm{l})$ e o valor de 48 horas após essa mesma sessão $(343,5 \pm 118,68 \mathrm{U} / \mathrm{l})$. O GP se comportou de maneira similar para esse marcador, apresentando diferença significativa $(p=0,02)$ apenas entre o valor basal da primeira sessão (83 $\pm 20,90 \mathrm{U} / \mathrm{l}$ ) e o valor obtido 48 horas após essa mesma sessão (253 $\pm 64,42 \mathrm{U} / \mathrm{l})$. A segunda sessão de EEM não causou alterações significativas na atividade sérica de CK para nenhum dos grupos. Ambos os grupos apresentaram FPs (GB $=99,1$ e GP $=134,4)$ e, quando comparados, os valores obtidos de FP não apresentaram diferença significativa. Os valores obtidos para a atividade sérica de CK estão representados graficamente na Figura 2.

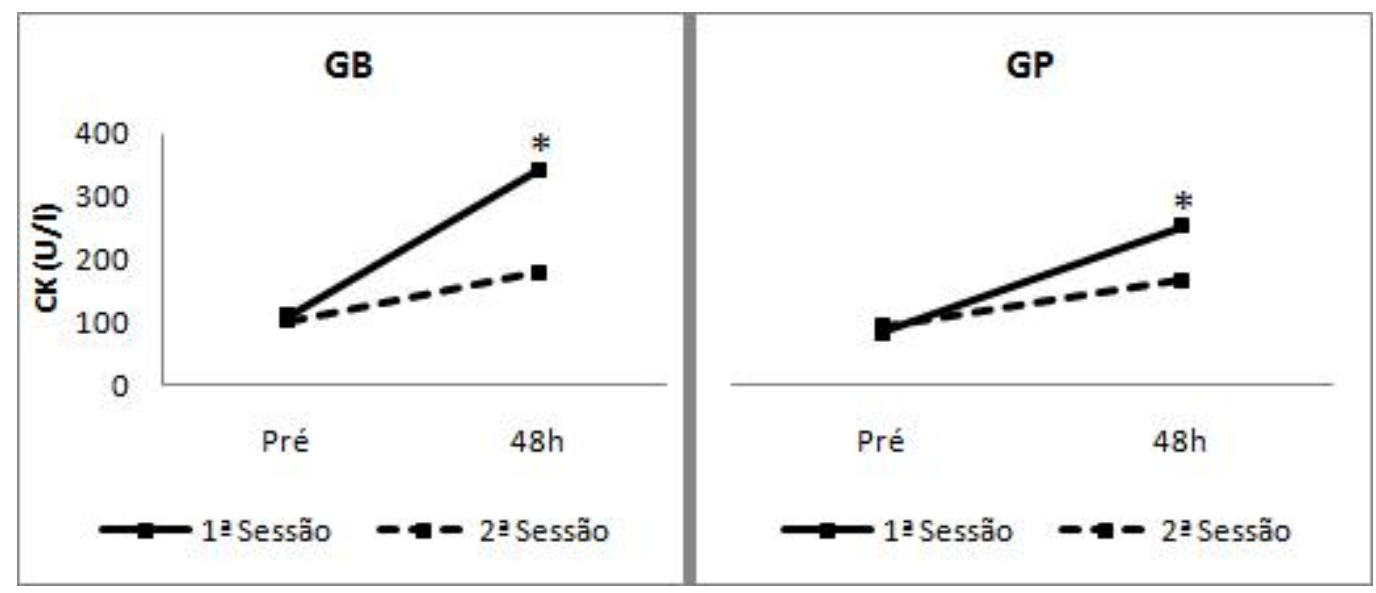

Figura 2. Mudanças na atividade sérica de CK antes e 48 horas após as 2 sessões de EEM para os grupos GB e GP. Os asteriscos $\left({ }^{*}\right)$ indicam diferença significativa $(p<0,05)$ em relação à condição basal da respectiva sessão. 


\section{Percepção subjetiva de dor}

Para o GB, foram encontradas diferenças significativas $(p=0,0003)$ entre os valores obtidos na condição basal referente à primeira sessão de EEM ( $3 \pm 1,8 \mathrm{~mm}$ ) e os valores encontrados na condição de 48 horas após essa mesma sessão $(51,8 \pm 12,17 \mathrm{~mm})$. Também foi encontrada diferença significativa $(p=0,02)$ entre a condição imediatamente após a primeira sessão de EEM $(21,1 \pm 10,36)$ e a condição de 48 horas após essa mesma sessão (51,8 $\pm 12,17 \mathrm{~mm})$. Baseando-se nesses dados pode-se concluir que houve FP para o GB. O GP apresentou aumentos

significativos ( $1^{\underline{a}}$ sessão: $p=0,001 ; 2^{\underline{a}}$ sessão: $p=$ $0,003)$ da PSD imediatamente após ambas as sessões de EEM (1 ${ }^{\text {a }}$ sessão: $45,6 \pm 11,35 \mathrm{~mm} ; 2^{\underline{a}}$ sessão: $47,3 \pm 5,73 \mathrm{~mm}$ ) em relação aos valores obtidos em suas respectivas condições basais (1 ${ }^{\text {a }}$ sessão: $2 \pm 0,98 \mathrm{~mm}$; $2^{\text {a }}$ sessão: $6,3 \pm 2,74 \mathrm{~mm}$ ). Os dados obtidos sugerem a não existência de um FP. Como não foi constatado FP para o GB, não foi rodado o teste de Mann-Whitney para a comparação de FPs. Os valores obtidos para a PSD estão representados graficamente na Figura 3.

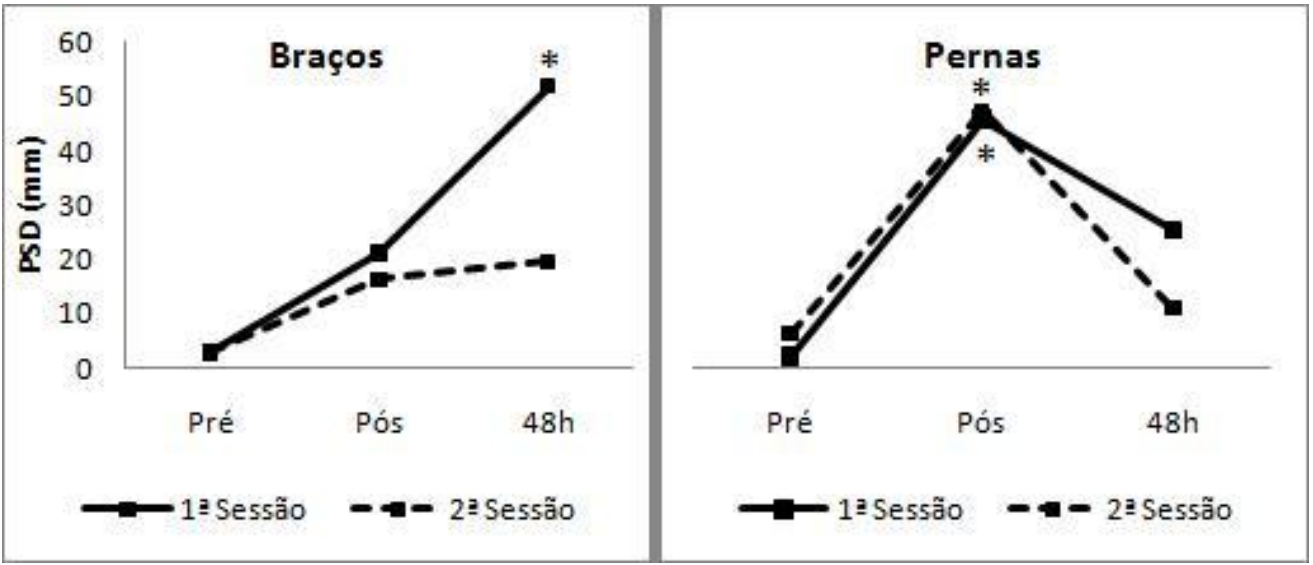

Figura 3. Mudanças na PSD antes, imediatamente depois e 48 horas após as 2 sessões de EEM para os grupos GB e GP. Os asteriscos $\left(^{*}\right)$ indicam diferença significativa $(p<0,05)$ em relação à condição basal da respectiva sessão.

\section{Pico de torque isométrico}

Para o GB, foram encontradas diminuições significativas $(p=0,003$ e 0,04$)$ nos valores de PTI imediatamente após a primeira $(44,1 \pm 3,8 \mathrm{Nm})$ e a segunda $(42,55 \pm 5,28 \mathrm{Nm})$ sessões de EEM em relação aos valores obtidos nas condições basais $(57,1 \pm 6,8 \mathrm{Nm}$ e $51,93 \pm 6,27 \mathrm{Nm}$, respectivamente). Porém, só foi identificada diferença significativa $(p=0,0007) 48$ horas após a primeira sessão de EEM $(42 \pm 4,9 \mathrm{Nm})$ em relação à condição basal. Esses dados indicam ter havido FP para o GB neste marcador. Já para o GP não foi encontrada diferença significativa entre os valores de PTI obtidos em nenhuma das condições após nenhuma das sessões de EEM, indicando inexistência de FP para este marcador no grupo GP. Os valores obtidos para o PTI estão representados graficamente na Figura 4.

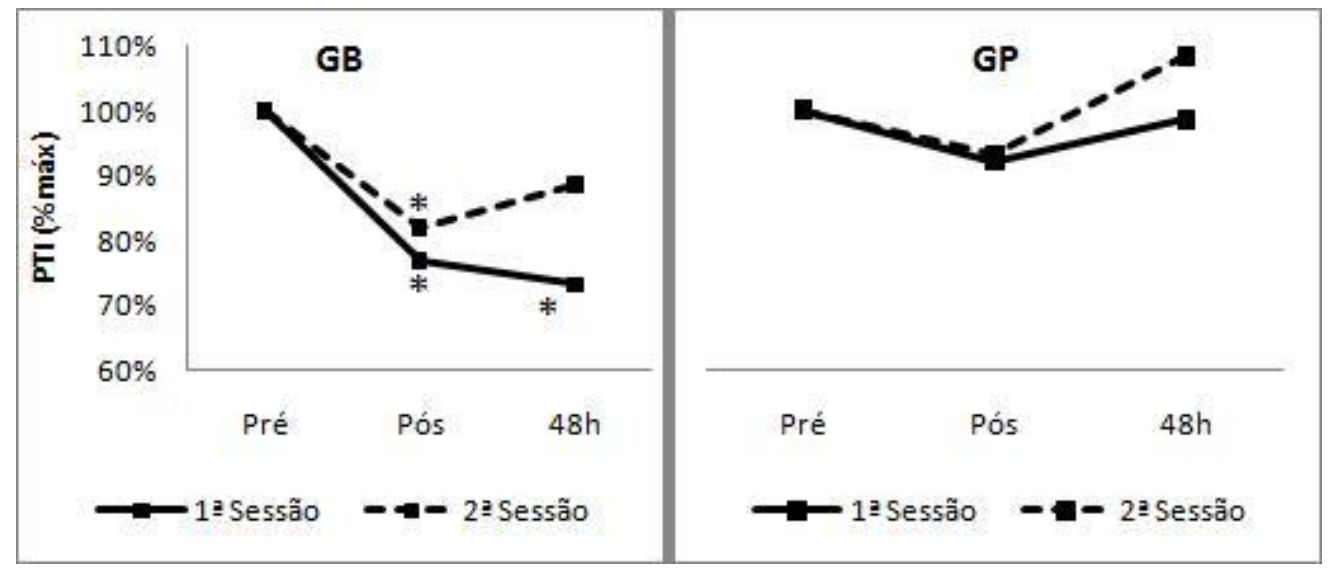

Figura 4. Mudanças no PTI antes, imediatamente depois e 48 horas após as 2 sessões de EEM para os grupos GB e GP. Os asteriscos $\left(^{*}\right)$ indicam diferença significativa $(p<0,05)$ em relação à condição basal da respectiva sessão. 


\section{Discussão}

O objetivo do presente estudo foi identificar se há diferenças entre o FP contra o DM conferido por uma sessão de EEM entre dois grupos musculares, testando a hipótese de que o FP seria menor para os EJ do que para os FC. Os grupos musculares e o critério de equalização da intensidade dos EEM foram adotados de acordo com estudos realizados anteriormente, que demonstraram diferenças na magnitude do DM para essa situação (JAMURTAS et al., 2005; SAKA et al., 2009; $\underline{\text { CHEN }}$ et al., 2011). A magnitude do DM encontrado no presente estudo foi consistente com a encontrada em outros estudos que utilizam os mesmos grupos musculares (ESTON et al., 1996; $\underline{\mathrm{CHEN}}$ et al., 2007; CHEN et al., 2011). Apenas um dos marcadores (CK) apresentou FP para ambos os grupos e, quando comparado, esse FP não apresentou diferença significativa. Para o PTI e a $P S D$, apenas o GB apresentou FP, indicando que, para esses marcadores, o FP é maior para o GB do que para o GP, confirmando a hipótese estabelecida.

Levando em consideração o que foi acima exposto, cabe discutir sobre as respostas individuais de cada um dos marcadores para os dois grupos, e a existência, ou não, do FP para esses grupos. Não há estudos de nosso conhecimento que tenham comparado os FP entre os grupos musculares analisados, ou quaisquer outros. $O$ que se encontra relativamente esclarecido na literatura foi exposto em três estudos. No primeiro deles, realizado por Jamurtas et al. (2005), concluiu-se que os FC apresentam DM de maior magnitude $e$ recuperação mais lenta, quando comparados aos EJ. O mesmo fenômeno foi confirmado posteriormente por Saka et al. (2009). Já Chen et al. (2011), realizaram um estudo comparando a magnitude e recuperação do DM não só para os FC e EJ, mas também para os EC e FJ. Com isso os autores concluíram que a magnitude $e$ recuperação do $D M$ foram semelhantes para os FC e EC, porém o mesmo não ocorre com os FJ e EJ. Os EJ foram o grupo menos acometido pelo DM e também apresentaram recuperação mais rápida, comparados com os demais. Todos os autores citados argumentaram que os EJ realizam maior número de ações excêntricas no dia a dia, quando comparados com os outros grupos estudados, e isso poderia conferir um FP parcial para essa musculatura, o que não ocorre para as demais.

Uma limitação a ser considerada tanto no presente estudo, quanto no que tem sido adotado na literatura, é o critério de controle de intensidade adotado na elaboração dos EEM. $O$ principal critério que tem sido utilizado leva em conta o tempo sob contração, ou seja, são adotadas amplitudes de movimento iguais, assim como velocidade angular, intervalo de recuperação e número de séries e repetições. Ao adotar este critério, o estímulo gerado pelas contrações excêntricas máximas pode sobrecarregar mais os músculos de menor AST (FC) do que os de maior AST (EJ), pela existência de maior número de fibras nestes, além de sua maior capacidade de produção de força. Uma alternativa à utilização do tempo sob contração como padrão de equiparação de intensidade seria o calculo do trabalho total realizado durante as sessões de EEM. Porém, se faz necessário um bom planejamento para que o trabalho contrátil a ser realizado nas séries de EEM não subestime o DM a ser causado nos músculos de maior AST nem superestime o mesmo para músculos de menor AST.

Outro aspecto a ser discutido como limitação no modelo de estudos que investigam o DM e o $E P$, são os marcadores indiretos adotados para a análise. $O$ presente estudo contou com três marcadores indiretos de DM (PTI, PSD e CK) por não termos ao nosso dispor o material e os recursos humanos necessários para a coleta de marcadores diretos, como análise in vitro de fibras musculares (obtidas por meio de biópsia) e imagens de ressonância magnética. Porém, aceitas nossas limitações, optamos pelos marcadores indiretos mais utilizados e recomendados dentro desta área de pesquisa (McHUGH, 2003; NOSAKA et al., 2005b; CHEN, 2011). Dos marcadores utilizados, o que apresenta maior reprodutibilidade e comportamento mais regular é o PTI, uma vez que as microlesões causadas no tecido muscular acometem mecanismos contráteis, diminuindo, assim, a capacidade de produção de força deste. Já os dois outros marcadores apresentam variações discrepantes entre os voluntários e até mesmo para um único voluntário.

A cinética da variação da concentração sérica de CK é dependente de fatores individuais de cada voluntário como estado de treinamento, 
metabolismo e, principalmente, atividade renal (BRANCACCIO et al, 2007). Porém, um fator que pode influenciar na resposta da CK é o volume muscular ou, mais especificamente, o número de fibras e/ou sarcômeros distribuídos ao longo do grupo muscular estudado. Quando se compara dois grupos musculares diferentes, é interessante um esforço para relativizar tanto as intervenções nesses músculos como também as medidas que serão realizadas na avaliação do DM neles induzido. Portanto, acreditamos que as medidas obtidas no presente estudo, e nos demais que verificam o DM, superestimem a magnitude do DM causado em músculos maiores ao introduzir apenas os valores de CK expressos em unidades por litro. Acreditamos que a divisão do valor de CK obtido por valores referentes à AST ou PTI represente melhor a proporção do DM causado tanto em grupos musculares com maior quantidade de fibras quanto em grupos menores.

Outro marcador utilizado no presente estudo foi a PSD. Nosso objetivo ao adotar tal medida era o de identificar a ocorrência do fenômeno denominado dor muscular de início tardio (DMIT), que costuma ocorrer cerca de 48 horas após uma sessão de exercícios intensos aos quais o corpo não está habituado (i.e., que causam DM) em decorrência de processo inflamatório (TRICOLI, 2001). Por mais consolidado que seja este marcador, a maneira com que se avalia a PSD é muito delicada e relativa. $O$ método de quantificação da PSD consiste na palpação do membro afetado pelo DM e posterior preenchimento de uma escala de $100 \mathrm{~mm}$ com análogos visuais em suas extremidades (McHUGH et al., 2003, SAKA et al., 2009, CHEN et al., 2011). Porém, esse tipo de mensuração é de caráter subjetivo, dependendo das experiências anteriores do voluntário e também de sua tolerância à dor (FOSCHINI et al., 2007). No caso de nosso estudo, acreditamos que os voluntários do GP podem ter confundido a sensação de dor muscular com a de fadiga localizada imediatamente após a sessão de exercícios, uma vez que o processo inflamatório só se manifesta por meio da DMIT a partir de oito horas após os EEM (ARMSTRONG, 1984; TRICOLI, 2001; FOSCHINI et al., 2007).

\section{Conclusão}

Com base nos dados obtidos, foi possível concluir que o DM conferido pelas sessões de EEM foi consistente com aquele encontrado na literatura geral, baseado nas respostas dos marcadores selecionados. As sessões de EEM conferiram FP para todos os marcadores coletados do GB enquanto apenas a CK apresentou FP no grupo GP. Portanto, podemos concluir que o FP foi maior, em geral, para os FC em comparação com os EJ, confirmando a hipótese previamente estabelecida. Esse fenômeno pode ter ocorrido em detrimento da existência de um FP prévio para os EJ, uma vez que estes realizam contrações excêntricas intensas com maior freqüência no dia-a-dia, quando comparados com os FC. Para futuros estudos comparando a magnitude do DM em diferentes grupos musculares, propomos que os valores obtidos de CK sejam relativizados de acordo com a AST ou PTI dos músculos danificados, para que a resposta deste marcador seja mais fidedigna com $o$ fenômeno que realmente ocorre no interior do tecido muscular, não abrindo espaço para questionamentos acerca de número de fibras e/ou volume muscular. Também sugerimos a utilização do trabalho contrátil realizado pelos músculos trabalhados como critério de igualdade de intensidade dos EEM, atentando para que não se superestime ou subestime os estímulos dos diferentes grupos musculares utilizados.

\section{Referências}

ARMSTRONG, R. B. Mechanisms of exerciseinduced delayed onset muscle soreness: a brief review. Medicine and Science in Sports and Exercise, Hagerstown, v. 16, p. 529-538, 1984.

BARASH, I. A.; PETERS, D.; FRIDEN, J.; LUTZ, G. J.; LIEBER, R. L. Desmin cytoskeletal modifications after a bout of eccentric exercise in the rat. American Journal of Physiology, Regulatory, Integrative and Comparative Physiology, Bethesda, v. 283, n. 4, p. 958-963, 2002.

BRANCACCIO, P.; MAFFULLI, N.; LIMONGELLI, F. M. Creatine kinase monitoring in sport medicine. British Medical Bulletin, Oxford, v. 81, p. 209-230, 2007.

BROCKETT, C. L.; MORGAN, D. L.; PROSKE, U. Human hamstring muscles adapt to eccentric exercise by changing optimum length. Medicine and Science in Sports and Exercise, Hagerstown, v. 33, n. 5, p. 783-790, 2001.

CHAPMAN, D.; NEWTON, M.; SACCO, P.; NOSAK, A. Greater muscle damage induced by fast versus slow velocity eccentric exercise. 
International Journal of Sports Medicine, New York, v. 27, p. 591-598, 2006.

CHEN, T. C.; NOSAKA, K.; SACCO, P. Intensity of eccentric exercise, shift of optimum angle, and the magnitude of repeated-bout effect. Journal of Applied Physiology, Bethesda, v. 102, p. 992999, 2007.

CHEN, T. C.; LIN, K.-Y.; CHEN, H.-L.; LIN, M.-J.; NOSAKA, K. Comparison in eccentric exerciseinduced muscle damage among four limb muscles. European Journal of Applied Physiology, Heidelberg, v. 111, p. 211-223, 2011.

CLARKSON, P. M.; HUBAL, M. J. Exerciseinduced muscle damage in humans. American Journal of Physical Medicine and Rehabilitation, Indianapolis, v. 81 (sup), p. s52s69, 2002. Suplemento.

CLARKSON, P. M.; NOSAKA, K.; BRAUN, B. Muscle function after exercise-induced muscle damage and rapid adaptation. Medicine and Science in Sports and Exercise, Hagerstown, v. 24, n. 5, p. 512-520, 1992.

CLARKSON, P. M.; TREMBLAY, I. Exerciseinduced muscle damage, repair, and adaptation in humans. Journal of Applied Physiology, Bethesda, v. 65, p. 1-6, 1988.

CONNOLLY, D. A. J.; McHUGH, M. P.; PADILLAZAKOUR, O. I. Efficacy of a tart cherry juice blend in preventing the symptoms of muscle damage.

British Journal of Sports Medicine, Londres, v. 40, p. 679-683, 2006.

DVIR, Z. Avaliações musculares, interpretações e aplicações clínicas. Barueri: Manole, 2002.

ESTON, R. G.; FINNEY, S.; BAKER, S.; BALTZOPOULOS, V. Muscle tenderness and peak torque changes after downhill running following a prior bout of isokinetic eccentric exercise. Journal of Sports Science, Walsall, v. 14, n. 4, p. 291-299, 1996.

FOSCHINI, D.; PRESTES, J.; CHARRO, M. A. Relação entre exercício físico, dano muscular e dor muscular de início tardio. Revista Brasileira de Cineantropometria e Desempenho Humano, Florianópolis, v. 9, n. 1, p. 101-106, 2007.

HOWATSON, G.; VAN SOMEREN, K.; HORTOBÁGYI, T. Repeated bout effect after maximal eccentric exercise. International Journal of Sports Medicine, New York, v. 28, p. 557-563, 2007.

JAMURTAS, A. Z.; THEOCHARIS, V.; TOFAS, T.; TSIOKANOS, A.; YFANTI, C.; PASCHALIS, V.;
KOUTEDAKIS, Y.; NOSAKA, K. Comparison between leg and arm eccentric exercises of the same relative intensity on indices of muscle damage. European Journal of Applied Physiology, Heidelberg, v. 95, p. 179-185, 2005.

LAPIER, T. K.; BURTON, H. W.; ALMON, R.; CERNY, F. Alterations in intramuscular connective tissue after limb casting affect contraction-induced muscle injury. Journal of Applied Physiology, Bethesda, v. 78, n. 3, p. 1065-1069, 1995.

LYNN, R.; MORGAN, D. L. Decline running produces more sarcomeres in rat vastus intermedius muscle fibers than does incline running. Journal of Applied Physiology, Bethesda, v. 77, n. 3, p. 1439-1444, 1994.

LYNN, R.; TALBOT, J. A.; MORGAN, D. L. Differences in rat skeletal muscles after incline and decline running. Journal of Applied Physiology, Bethesda, v. 85, n. 1, p. 98-104, 1998.

MALM, C.; LENKEI, R.; JÖDIN, B. Effects of eccentric exercise on the immune system in men. Journal of Applied Physiology, Bethesda, v. 86, p. 461-468, 2002.

MAYHEW, D. L.; THYFAULT, J. P.; KOCH, A. J. Rest-interval length affects leukocyte levels during heavy resistance exercise. Journal of strength and conditioning research, Colorado Springs, v. 19, n. 1, p. 16-22, 2005.

McHUGH, M. P. Recent advances in the understanding of the repeated bout effect: the protective effect against muscle damage from a single bout of eccentric exercise. Scandinavian Journal of Medicine and Science in Sports, Copenhagen, v. 13, p. 88-97, 2003.

McHUGH, M. P.; CONNOLLY, D. A. J.; ESTON, R. G.; GARTMAN, E. J.; GLEIM, G. W.

Electromyographic analysis of repeated bouts of eccentric exercise. Journal of Sports Sciences, Walsall, v. 19, p. 163-170, 2001.

NOSAKA, K.; NEWTON, M.; SACCO, P. Attenuation of protective effect against eccentric exercise-induced muscle damage. Canadian Journal of Applied Physiology, Ottawa, v. 30, n. 5, p. 529-542, 2005a.

NOSAKA, K.; NEWTON, M.; SACCO, P. Partial protection against muscle damage by eccentric actions at short muscle lengths. Medicine and Science in Sports and Exercise, Hagerstown, v. 37 , n. 5, p. 746-753, 2005b.

NOSAKA, K.; SAKAMOTO, K. Effect of elbow joint angle on the magnitude of muscle damage to the elbow flexors. Medicine and Science in Sports 
and Exercise, Hagerstown, v. 33, n. 1, p. 22-29, 2001.

NOSAKA, K.; SAKAMOTO, K.; NEWTON, M. How long does the protective effect on eccentric exercise-induced muscle damage last? Medicine and Science in Sports and Exercise, Hagerstown, v. 33, n. 9, p. 1490-1495, 2001.

NOSAKA, K.; SAKAMOTO, K.; NEWTON, M.; SACCO, $P$. The repeated bout effect of reducedload eccentric exercise on elbow flexor muscle damage. European Journal of Applied

Physiology, Heidelberg, v. 85, p. 34-40, 2001.

NOSAKA, K.; MUTHALIB, M.; LAVENDER, A.; LAURSEN, P. B. Attenuation of muscle damage by preconditioning with muscle hyperthermia 1 day prior to eccentric exercise. European Journal of Applied Physiology, Heidelberg, v. 99, p. 183192, 2007.

POUSSON, M.; VAN HOECKE, J.; GOUBEL, F. Changes in elastic characteristics of human muscle induced by eccentric exercise. Journal of Biomechanics, Atlanta, v. 23, n. 4, p. 343-348, 1990.

SAKA, T.; AKOVA, B.; GUR, H.; OZARDA, Y.; SEKIR, U.; YAZIC, Z. Difference in the magnitude of muscle damage between elbow flexors and knee extensors eccentric exercises. Journal of Sports Science and Medicine, Ankara, v. 8, p. 107-115, 2009.

TRICOLI, V. Mecanismos envolvidos na etiologia da dor muscular tardia. Revista Brasileira de Ciência e Movimento, Brasília, v. 9, n. 2, p. 3944, 2001.

WARREN, G. L. et al. Decreased EMG median frequency during a second bout of eccentric contractions. Medicine and Science in Sports and Exercise, Hagerstown, v. 32, n. 4, p. 820829, 2000.
Endereço:

Leonardo Coelho Rabello de Lima

Avenida 24-A, 1515 Bela Vista

Rio Claro SP Brasil

13506-900

Telefone: (19) 3526.4320

Fax: (19) 3526.4321

e-mail: leonardocrlima@gmail.com

Recebido em: 13 de março de 2011. Aceito em: 29 de maio de 2011.

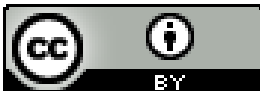

Motriz. Revista de Educação Física. UNESP, Rio Claro, SP, Brasil - elSSN: 1980-6574 - está licenciada sob Creative Commons - Atribuicão 3.0 